\author{
Hansueli Albonico \\ Danielle Lemann
}

Wegleitend dabei wurde alsbald die Frage, wie unsere ägyptischen Kollegen und Kolleginnen zur Begegnung mit einer Medizin kommen können, die versucht, ein Gleichgewicht zwischen materiellen und spirituellen Aspekten von Gesundheit und Krankheit herzustellen. Unsere moderne westliche «Schul»-Medizin ist ja aus der arabischen Medizin hervorgegangen. Die Schulmedizin ist «ihre Medizin»; sie scheinen kein Bedürfnis nach einer Komplementärmedizin zu haben. Der Koran böte, wie auch das Neue Testament, an sich reichlich Materialien für die Bewusstwerdung, dass jede Arzt-Patienten-Begegnung immer auch spirituelle Dimensionen beinhaltet. Nur ist die Schulung dieses Bewusstseins hüben und drüben weitgehend verloren gegangen.

In Zusammenarbeit mit dem langjährigen Mentor des Medical Centers (Abb. 1-4), Dr. Hans Werner, Oeschelbronn, entschlossen wir uns deshalb zur Einführung eines regelmässigen Ärzteseminars in anthroposophischer Medizin, welches im März 2006 begann und seither als ein- bis dreitägiger Kurs achtmal durchgeführt wurde. Als Inhaberin eines Masters of Medical Education versuchte Danielle Lemann, die Seminarien zunehmend interaktiv, mit Gruppenarbeit, Flüstergruppen und "problem-based learning» zu führen, was sich, da noch völlig ungewohnt, als erstaunliche Herausforderung erwies. Auch das Instrument der kontinuierlichen Seminarevaluation war überraschend neu.

Thematisch versuchten wir, an die alltäglichen Patientenbegegnungen im Medical Center anzuknüpfen.

\title{
Sanfte Medizin für das Land am Nil - Komplementärmedizin in Ägypten
}

\section{Ein Beitrag zum Dialog zwischen Islam und westlicher Welt}

Seit 1977 gibt es in Ägypten auf Initiative von Dr. Ibrahim Abouleish, Träger des alternativen Nobelpreises und Teilnehmer am WEF (World Economic Forum), das Farmprojekt SEKEM (www.sekem.com), welches mit den Mitteln der biodynamischen Landwirtschaft die Wüste in ein blühendes Paradies verwandelt hat. Mehr als 1000 Menschen arbeiten direkt auf dem Betrieb, weitere 800 Bauernfamilien im ganzen Niltal haben sich dem Projekt angeschlossen. Mittlerweile gibt es in SEKEM Heilpflanzenanbau und -verarbeitung, Textilverarbeitung, Schulen, heilpädagogische Werkstätten und ein Medizinisches Zentrum, welches für die gesamte Region zur Verfügung steht. Meine Frau, Danielle Lemann, und ich wurden eingeladen, in diesem Zentrum an einer angepassten anthroposophisch-medizinischen Betreuung der Bevölkerung zu arbeiten.

Dabei zeigte sich z.B., dass es für die ägyptischen Kollegen fast undenkbar ist, ein Kind mit Fieber nicht sofort mit fiebersenkenden Mitteln und Antibiotika zu behandeln; oft werden schon bei banalen Erkältungen Antibiotika-Infusionen verabreicht. Diese «Schnellschuss»Medizin hat aber auch in Ägypten Nachteile: Die zur nachhaltigen Gesundheitsentwicklung notwendige Auseinandersetzung des Immunsystems mit der Umwelt wird unter- bunden, Immun- und Autoimmunkrankheiten nehmen sprunghaft zu.

Ägypten hat eine enorm hohe Prävalenz von Hepatitis-C-Infektionen, zum grossen Teil als Folge früherer Bilharziose-Behandlungen mit Mehrfachgebrauch von Spritzen. Interferone und Ribavirin sind für den grössten Teilder Bevölkerung unerschwinglich. Hier liegt noch ein grosses Feld für vergleichsweise billige Behandlungen mit pflanzlichen Heilmitteln. In der Behandlung von Hepatomen wie auch
Abb. 1. Team Medical Center, mit Dr. Ibrahim Abouleish (Mitte, mit Halstuch).

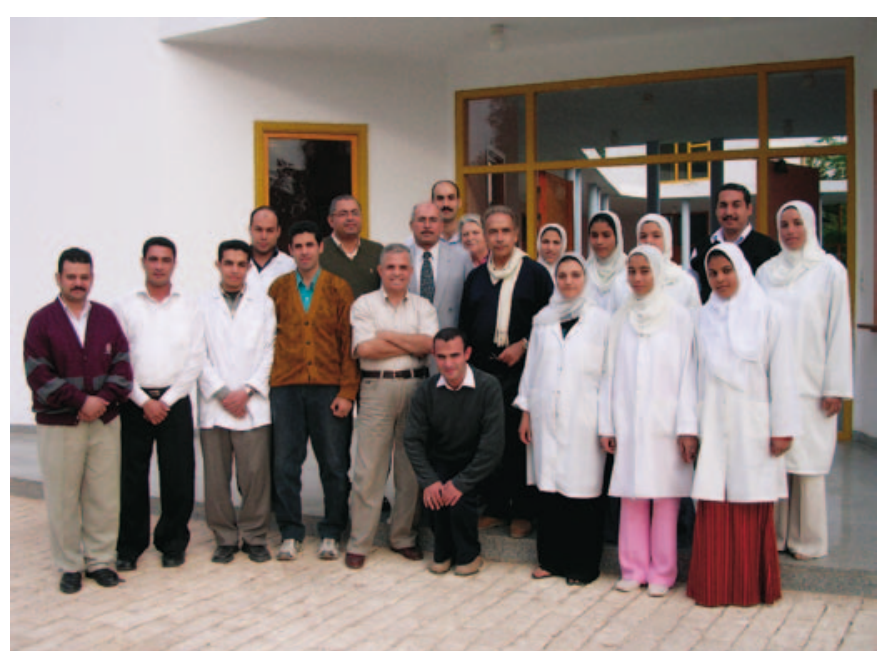

\section{KARGER}

Fax +4976145207 14 Information@Karger.de www.karger.com
(C) 2010 S. Karger GmbH, Freiburg

Accessible online at:

www.karger.com/szg
Dr. med. Hansueli Albonico

Komplementärmedizinische Abteilung

Regionalspital Emmental

Dorfbergstrasse 30, 3550 Langnau, Schweiz

Tel. +41344213131

hansueli.albonico@rs-e.ch 
Mamma- und Blasenkarzinomen hat sich die Mistelbehandlung auch in Studien [1-3] als wesentlich wirksamer als in Europa erwiesen.

Die Medikamente der konventionellen «Schulmedizin» werden fast ausschliesslich synthetisch hergestellt; sie stammen also alle aus Erdöl. Demgegenüber weist der Koran gleichermassen wie etwa Paracelsus darauf hin, dass dem Arzt für seine Heilbemühung eigentlich die ganze Natur zur Verfügung stehen sollte! Darüber hinaus gibt es eine breite $\mathrm{Pa}$ lette künstlerischer Therapien. Damit kann der Arzt/die Ärztin die Krank-

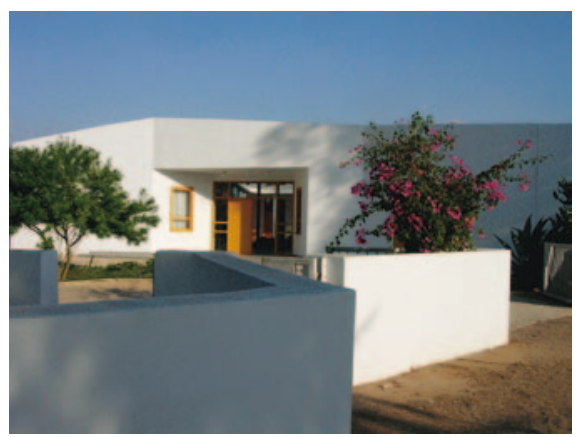

Abb. 2. Eingang zum Medical Center.

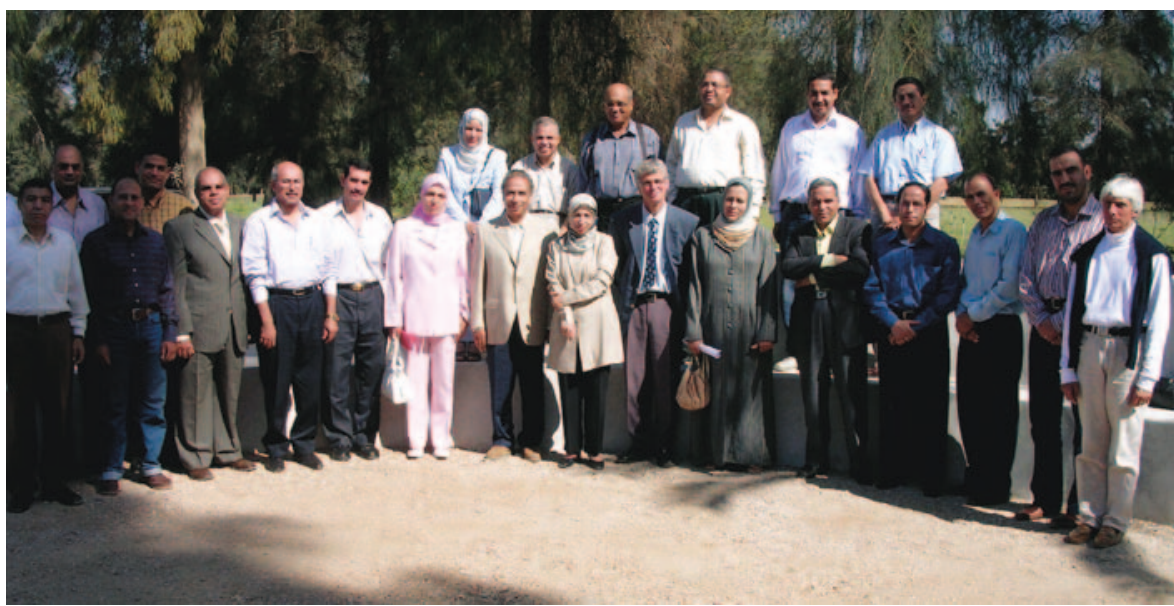

heiten individueller und schonender behandeln. Die Verantwortung ist dabei eine dreifache: Nebst dem Bewusstsein, dass Heilbemühungen Auswirkungen auf die ganze Biografie des Patienten haben, muss der Arzt/ die Ärztin eine Wahrnehmung für das soziale Umfeld des Patienten entwickeln und dabei immer auch den Umweltbezug berücksichtigen.

Eine zentrale Bedeutung kommt auch in Ägypten - der Ernährung zu. Der Patient muss nicht nur genügend Nährstoffe einnehmen, sondern gleichermaßen genügend Lebenskräfte. Gerade z.B. bei den vielen Patienten

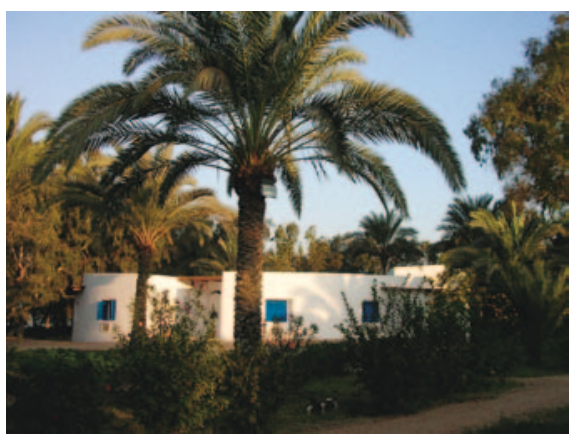

Abb. 3. Mahad-Seminarzentrum.

\section{Literatur}

1 Mabed M, El-Helw L, Sharma S: Phase II study of viscum fraxini-2 in patients with advanced hepatocellular carcinoma. $\mathrm{Br} \mathrm{J}$ Cancer 2004;90:65-69.

2 Mahfouz M, Ghaleb A, Hamza M, Fares L, Moussa L, Saed S, Fouad F, Tony O, Tohamy A: Multicenter open labelled clinical study in advanced breast cancer patients. J Egypt Nat Canc Inst 1999;11:221-227.

3 Saied W, Shamaa S, Mabed M: Chemotherapy of hepatocellular carcinoma. Thesis. Mansourana University, Faculty of Medicine, 2003.

Abb. 4. Teilnehmer Ärzteseminar 2008 mit Dr. Ibrahim Abouleish (Mitte vorne). 